\title{
Der Teufel und die Details
}

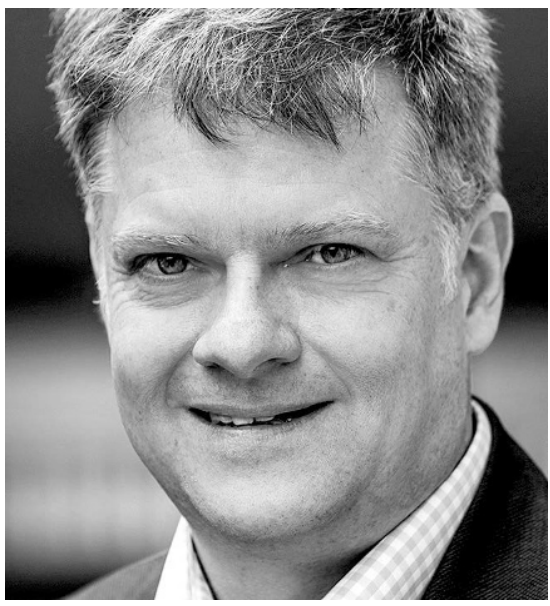

Seit gut sieben Monaten ist die - für viele Unternehmen ganz überraschend - am 25. Mai 2018 in Kraft getretene Datenschutz-Grundverordnung (DSGVO) nun geltendes Recht. Und auch wenn sie in wesentlichen Teilen das bewährte Bundesdatenschutzgesetz lediglich fortschreibt, so enthält sie doch die eine oder andere Tücke. Befeuert vom Medienrummel, von dem das Inkrafttreten der DSGVO begleitet wurde (und der dem Datenschutz wahrscheinlich mehr geschadet als genutzt haben dürfte), wurde in zahlreichen Unternehmen die Umsetzung der vom BDSG (alt) abweichenden Bestimmungen in Angriff genommen. Und dabei eröffnen sich unerwartete Flanken: Viele der mit der Umsetzung verbundenen Unwägbarkeiten sind nicht den Bestimmungen der DSGVO an sich zuzuschreiben, sondern der Tatsache, dass sich ein gemeinsames Verständnis für die Umsetzungspraxis eines (neuen oder geänderten) Gesetzes erst mit der Zeit entwickelt. In diesem Heft ziehen wir mit der Betrachtung ausgewählter Umsetzungsaspekte ein Zwischenfazit.

Buchmann und Eichhorn untersuchten kurz nach Inkrafttreten der DSGVO die Umsetzung der Auskunftspflicht gegenüber Betroffenen (Ar. 15 DSGVO) bei großen Online-Händlern und Dienstleistungsunternehmen - mit überraschenden Ergebnissen. Insbesondere die Identitätsprüfung bei elektronischen Auskunftsersuchen Betroffener kann eine Herausforderung sein, wenn sie den Betroffenen keine inakzeptablen Hürden aufbürden soll: Petrlic stellt verschiedene Verfahren gegenüber und vergleicht ihre Eignung aus Sicherheits- und Benutzerperspektive.

Der Verschlüsslung von Daten kommt mit der DSGVO durch die explizite Erwähnung eine besondere Bedeutung zu. Ob das nun technisch gerechtfertigt ist oder nicht - geeignete Datenschutz- und Datensicherheitskonzepte müssen diesem Punkt Rechnung tragen. Die DSGVO lässt allerdings offen, ob auch eine Transportverschlüsselung, die vollkommen transparent für den Nutzer erfolgt, der Anforderung genügt. Kneuper und Macke gehen dieser Frage im Zusammenhang mit der Verschlüsselung von E-Mail-Nachrichten (und deren Anhängen) nach.

Webseitenbetreiber sind nach der - umstrittenen und technisch veralteten - „Cookie-Richtlinie" (RL 2009/136/EG) aus dem Jahr 2009, die ursprünglich zeitgleich mit dem Inkrafttreten der DSGVO durch eine neue E-Privacy-Verordnung ersetzt werden sollte, verpflichtet, das Einverständnis des Seitenbenutzers für die Speicherung von Nutzungsdaten in "Cookies" einzuholen. Kulyk, Gerber, Volkamer und Hilt haben nun in einer Nutzerstudie untersucht, welchen Einfluss ein solcher Hinweis, abhängig von der Formulierung, auf das weitere Verhalten der Seitenbesucher hat.

Angesichts der signifikant erhöhten Maximalbußgelder und, wichtiger noch, der Verpflichtung der Aufsichtsbehörden, dass verhängte Geldbußen „in jedem Einzelfall wirksam, verhältnismäßig und abschreckend" sind (Art. 83 DSGVO), steigt auch die Befürchtung bei Datenschutzbeauftragten, im Falle der Verhängung eines Bußgelds vom Unternehmen in Regress genommen zu werden - denn nach Art. 39 DSGVO wirkt der Datenschutzbeauftragte nicht mehr nur auf die Umsetzung des Datenschutzes im Unternehmen oder der Behörde hin, sondern es obliegt ihm die „Überwachung der Einhaltung dieser Verordnung". Da liegt der Abschluss einer Haftpflichtversicherung nahe: Breyer und Duensing haben eine Marktsichtung geeigneter Angebote vorgenommen und stellen sie in ihrem Beitrag gegenüber.

Die Beiträge zeigen, dass es für zahlreiche Detailfragen der DSGVO-Umsetzung bisher keine eindeutigen Antworten oder Umsetzungsempfehlungen gibt - eine Beobachtung, die die Entwicklung und Veröffentlichung einheitlicher Vorstellungen der Aufsichtsbehörden zur Anwendung der DSGVO umso dringlicher macht.

\section{Dirk Fox}

